\title{
BiLeSA: Mathematik mit digitalen Bildern sichtbar machen
}

\author{
Rebekka Axthelm
}

Die Frage „Wozu braucht man das?“ vonseiten der Studierenden oder Aussagen wie „Das habe ich im Beruf später nie mehr benötigt.“ von ehemaligen Studierenden ist den meisten Mathematikdozierenden sehr vertraut. Stets begegnet uns der Wunsch „,in der Lehre die Anwendungsrelevanz aufzuzeigen“ vonseiten der Kollegen. Wo liegt die Schwierigkeit? Oft ist der Weg von der ersten mathematischen Formulierung bis zu einem wirklich interessanten, praxisnahen Beispiel sehr weit. Das fordert die Geduld der Lernenden. Und ist man endlich angekommen, haben sich so viele Lücken auf dem Weg angehäuft, dass das Verständnis für das Gesamte nicht mehr aufgebracht werden kann. Nur wenige „sehen“ das zugrunde liegende Konzept noch.

Das Projekt BiLeSA beinhaltet zwei Teilprojekte, die sich jeweils mit einem Thema aus den Grundvorlesungen zur Mathematik an den Hochschulen ZHAW und HTWG befassen. Im Teilprojekt BiLeSA $A_{\text {LIN }}$ werden lineare und affin lineare Abbildungen behandelt und im Teilprojekt BiLeSAPDE, welches in diesem Kapitel behandelt wird, sind Ableitungsterme für multivariate Funktionen und Diffussionsprozesse Thema. Nach Ende des Projekts wurde BiLeSA COM $_{\text {um das }}$ Paket BiLeSA erweitert, welches die Veranschaulichung der Potenz komplexer Zahlen beinhaltet. Abb. 6.1 zeigt eine grobe Übersicht der Funktionalität der App.

R. Axthelm (凶)

Fakultät Informatik, (HTWG) Hochschule für Technik, Wirtschaft und Gestaltung

Konstanz, Konstanz, Deutschland

E-Mail: rebekka.axthelm@htwg-konstanz.de

(C) Der/die Autor(en) 2022 


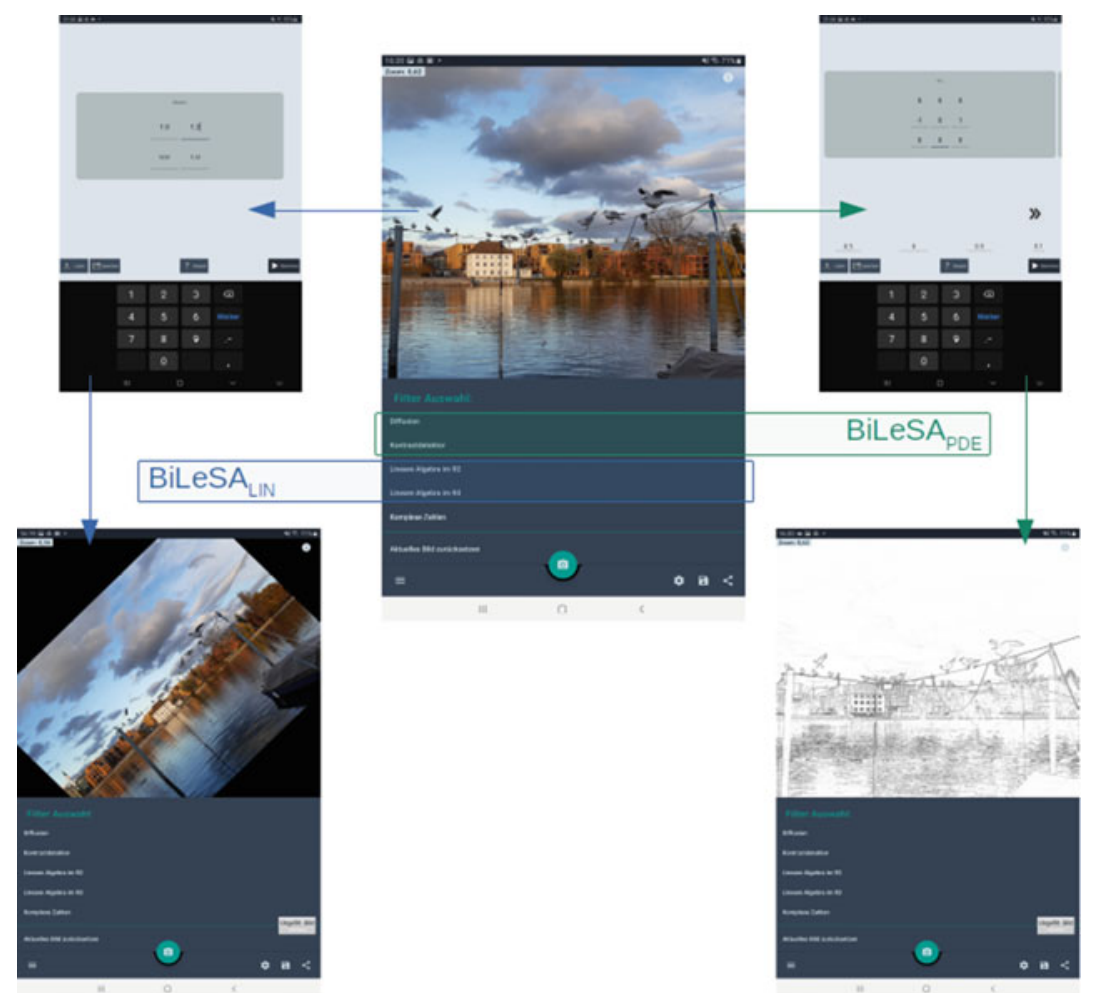

Abb.6.1 Übersicht der App-Funktionalität für die BiLeSA-Lernobjekte

\subsection{Ausgangssituation}

Wir wollen uns im Projekt BiLeSA dem Bruch ,Theorie-Praxis“ widmen, mit dem Ziel, die Brücke zwischen Theorie und Praxis so zu gestalten, dass die Neugierde auf das Relevante nicht verloren geht. „Mathematik sehen“, „,mathematische Prozessbeschreibung verstehen“, „Lernen durch Erfahren“ sind die Zielsetzungen von BiLeSA. Wir machen uns dabei die Möglichkeiten der Bildverarbeitung zunutze, um Methoden der Mathematik sichtbar zu machen. Zu diesem Zweck wurde 
eigens eine App ${ }^{1}$ entwickelt. Der didaktische Mehrwert besteht darin, dass die betrachteten Prozesse nicht einfach per Klick in sinnvoller Weise ausgeführt werden können, sondern dass zur Bedienung zunächst mathematische Kenntnisse erarbeitet werden müssen. Das führt einerseits zu einer gesteigerten intrinsischen Motivation der Lernenden, sich mit den Lehrinhalten zu befassen und andererseits bietet es die Möglichkeit, in einen von vielen Anwendungsbereichen Einblick zu gewähren.

Wir betrachten eine zweistündige Analysisvorlesung (im Hörsaal) mit zwei Übungsstunden (im Seminarraum) und einer Laborstunde (im Rechnerraum) pro Woche. Die Teilnehmenden sind Studierende der HTWG im Studiengang Angewandten Informatik im zweiten Semester. Einige haben Abitur, wovon aber grundsätzlich nicht ausgegangen werden kann. Die Größe der Hörerschaft wechselt zwischen ungefähr 30 und ungefähr 60 Personen. Das Projekt BiLeSAPDE befasst sich mit einem Kapitel der Vorlesung, nämlich mit „Ableitungen in höheren Raumdimensionen“. Damit sind Ableitungen von sowohl multivariaten als auch vektorwertigen Funktionen und Kombinationen daraus, also Funktionen $\mathrm{u}$, mit $\mathrm{u}: \mathrm{R}^{\mathrm{n}} \rightarrow \mathrm{R}^{\mathrm{m}}$ gemeint. Die Lernziele in diesem Kapitel umfassen das Kennen und Aufstellenkönnen von verschiedenen Ableitungstermen wie Laplace-Operator, Hesse- und Jacobi-Matrix, Gradient, Richtungsableitungen erster und zweiter Ordnung mit konstanten und auch variablen Richtungen. All diese Ableitungsterme basieren auf sogenannten partiellen Ableitungen. Bis hierhin ist der Unterrichtsinhalt sehr abstrakt und zeigt keinen Bezug zur Praxis. Studierende empfinden diesen Unterrichtsteil eher uninteressant. Und das, obwohl wir allein mit diesen wenigen Termen bereits sehr viele Prozesse unserer Welt beschreiben können. Prozesse, die wir in dieser Lehreinheit besprechen sind isotrope und anisotrope Diffusion im Hinblick auf die Anwendung in der digitalen Bildverarbeitung. Als einfaches Beispiel kann man sich ein Weichzeichnen oder Schärfen, mit und ohne Vorzugsrichtung, von digitalen Bildern vorstellen. Der oben beschriebene lange Weg von den Formeln bis zur Anwendungsrelevanz beschreibt damit einen besonders ausgedehnten Spagat zwischen spröder, abstrakter Theorie und spannenden Phänomenen, die gerade für angehende Informatiker und Informatikerinnen besonders interessant sind. An genau dieser Stelle adressieren wir den Bruch zwischen Theorie und Praxis.

Ziel des Projekts ist es, diese Lehreinheit so zu konzipieren, dass Lernende und Lehrende zwischen praktischen Anwendungen sowie Sichtbarmachen der Ableitungsterme und rein abstrakter Rechenmethoden auf vielfältige Weise balancieren

${ }^{1}$ BiLeSA App auf https://www.ios.htwg-konstanz.de/modelling-and-simulation-workgroup. 
können. Die didaktischen Leitprinzipien von BiLeSA sind „Lernen durch Erfahren“ und „Selbstreguliertes Lernen“. Dabei soll die Komponente des Erfahrens nicht erst am Ende einer langen „Durststrecke“ einsetzen sondern sich in möglichst kurzen Abständen mit dem Klären der Rechenmethoden abwechseln. Wie oft der Wechsel zwischen „Theorie“ und „Praxis“" stattfindet kann ,selbstreguliert“ gestaltet werden.

Obwohl zur Veranstaltung eine gesonderte Laborstunde zur Verfügung steht, die Raum gibt für mathematisches Rechnen am Computer, würde ein selbstständiges Programmieren einen zu großen Zeitaufwand bedeuten. Daher wurde, speziell für dieses Kapitel, eine Smartphone App entwickelt, die es erlaubt, den praktischen Teil in kürzerer Zeit umzusetzen. Die Idee der App ist, dass sie die numerischen Berechnungen umsetzt, aber nur mit erreichten Lernzielen bedient werden kann.

\subsection{Konzept}

BiLeSAPDE beinhaltet darüberhinaus ein spezielles Unterrichtskonzept, das sowohl für Lehrende als auch Lernende Variationen der Themenerarbeitung zulässt. Die spezielle Konzeptionierung baut auf einer Zusammenstellung in Matrixstruktur der Einzelthemen auf und trägt keinen in der Didaktik bekannten Namen. Sie ist im Laufe des Projekts, basierend auf den Unterrichtserfahrungen, den Evaluationsergebnissen und der fachlichen Unterstützung vonseiten des Basisprojekts im IBH-Lab „Seamless Learning“, entstanden. Sowohl das Selbststudium als auch das Lehrkonzept kann nach verschiedenen Gesichtspunkten gestaltet werden:

- In der klassischen Reihung, wie sie in der Mathematik üblich ist, werden zunächst alle theoretischen Begriffe, Formeln und Rechenmethoden besprochen, und am Ende wird mit Beispielen die Anwendungsrelevanz erklärt.

- Die Matrixstruktur, auf die später noch näher eingegangen wird, ermöglicht einen kreativen und abwechslungsreichen Unterrichtsablauf, in dem auf verschiedene Weise ein Mischen von Theorie und Anwendungsbeispielen möglich ist.

In der klassischen Vorgehensweise werden zunächst die Rechenmethoden der Ableitungsterme besprochen und die Anwendung auf kontinuierliche Funktionen geübt. Im Anschluss leitet man die mathematische Beschreibung, also Modelle, 


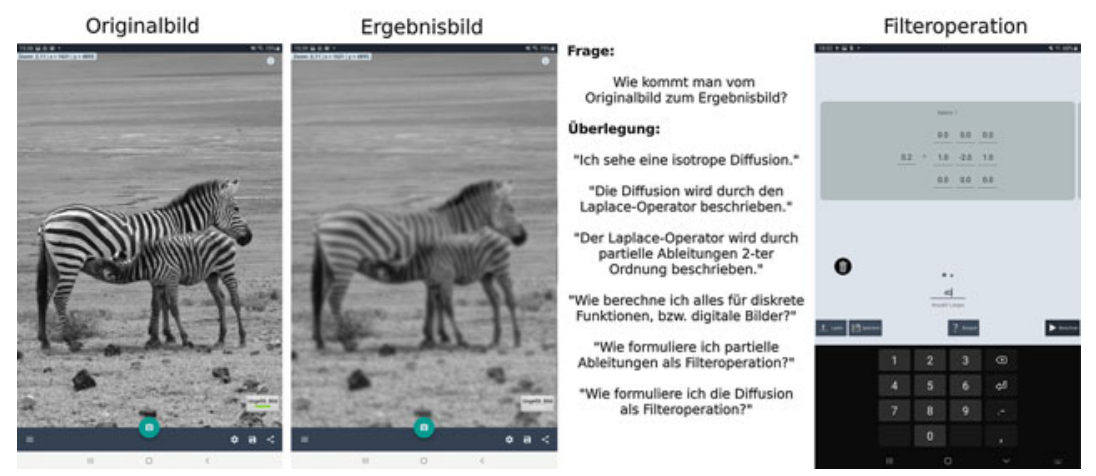

Abb. 6.2 Beispiel einer Aufgabenstellung unter Verwendung der BiLeSA-App, die ein Hinterfragen der Lernziele erfordert

von diffusiven Prozessen her. Digitale Bilder können als diskrete Funktionen interpretiert werden. Um diffusive Prozesse auf digitale Bilder anwenden zu können, müssen alle Ableitungsterme und Modelle speziell für diskrete Funktionen hergeleitet werden. Eine in der Praxis typische lineare Operation auf digitale Bilder wird über diskrete Faltungen ausgeübt, woraus bestimmte Filterdarstellungen, wie sie in der Bildverarbeitung üblich sind, resultieren. $\mathrm{Zu}$ jedem diffusiven Prozess passt am Ende eine Linearkombination bestimmter Filter. Bis hierher wird abstrakt mit Bleistift und Papier gearbeitet. Erst mit der Kenntnis der Filter kann nun die App bedient werden. Abb. 6.2 zeigt ein Beispiel.

Es soll interpretiert werden welcher Prozess vom Originalbild zu dem weichgezeichneten Ergebnisbild führt und mit welchen Ableitungstermen dieser Prozess mathematisch beschrieben wird. Dann müssen die Ableitungsterme für diskrete Funktionen, als was digitale Bilder verstanden werden können, aufgestellt werden. Eine anschließende Umformulierung in eine Filteroperation ermöglicht die Bedienung der App. Studierende können dann direkt prüfen, ob Sie mit ihrem Ergebnis das vorgegebene Ergebnisbild reproduzieren können.

Die Einzelkomponenten der Lehreinheit lassen sich in einer Matrixstruktur zusammenstellen (s. Abb. 6.3). Die Anordnung wie sie in Abb. 6.4 dargestellt ist zeigt eine vertikale Ausrichtung der verschiedenen Ableitungsterme von erster Ordnung (mit Richtungsableitung) über zweiter Ordnung, Richtungsableitung zweiter Ordnung mit konstanter Richtung und letztlich auch mit variabler Richtung. In horizontaler Richtung sind die Themen nach den Kategorien kontinuierliche Ableitungsterme, kontinuierliche Modelle, diskrete Funktionen, diskrete 


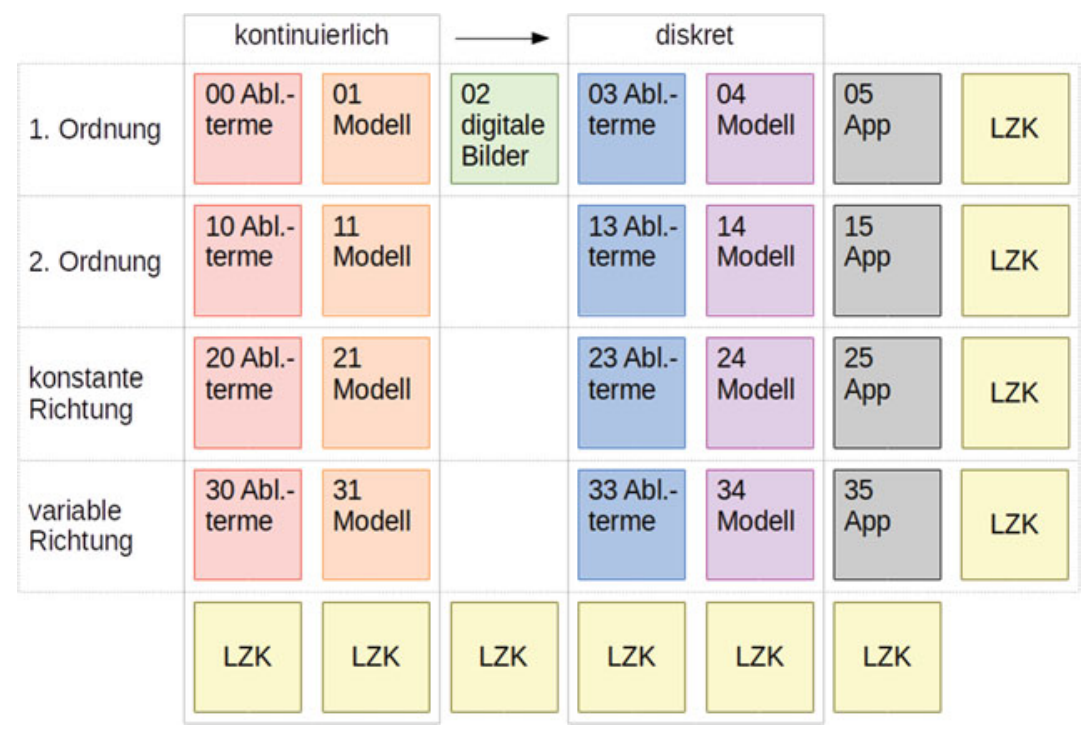

Abb. 6.3 Themenanordnung in Matrixstruktur. (LZK = Lernzielkontrolle)

Ableitungsterme und diskrete Modelle, sowie letztlich Aufgabenstellungen für die App sortiert.

„Selbstreguliertes Lernen“ bedeutet in diesem Kontext, dass es nicht den einen roten Faden gibt, sondern, dass es verschiedene Wege gibt, die durch den zu erarbeitenden Stoff leiten. Manche Wege sind schnell durchgearbeitet, andere gestalten sich abwechslungsreich und sind daher eher interessant. Der Lernweg für das Selbststudium kann individuell gewählt werden. Genauso variabel ist auch die Durchführung des gesamten Unterrichts, also Vorlesung, Übung und Praktikum, bez. Labor. Abb. 6.4 zeigt Beispiele von möglichen Wegen durch die Inhalte. Ein spaltenweiser Aufbau der Lerninhalte entspricht dem klassischen Weg, wie oben beschrieben; schnell und pragmatisch. Dagegen lässt sich durch den zeilenweisen Aufbau eine bessere Durchmischung von „Theorie und Praxis“ erreichen, womit Lernziele auf eine verstärkt experimentelle Weise erreicht werden können, da nach kleinen Schritten in der Theorie jeweils Anreize zu weiteren Schritten geschaffen werden. Der Nachteil ist der benötigte größere Zeitaufwand, wie er unter Umständen nicht zur Verfügung steht. Es lassen sich verschiedene Kompromisse zwischen interessantem Unterricht und zügigem Vorankommen durch eine blockweise Gestaltung erreichen. Wichtig ist nur von oben nach unten und von links nach rechts vorzugehen; auch innerhalb der Blöcke. 


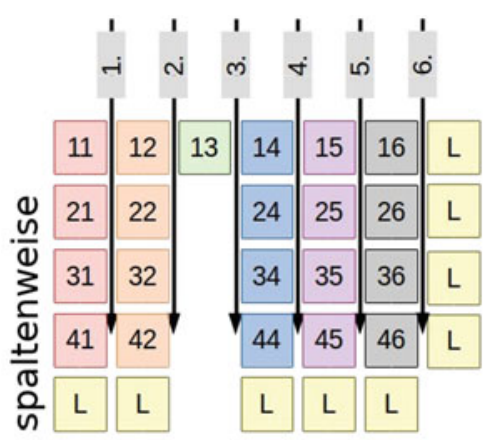

blockweise

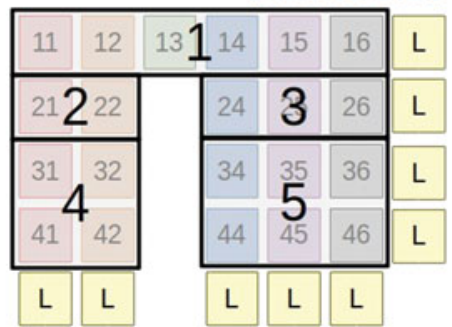

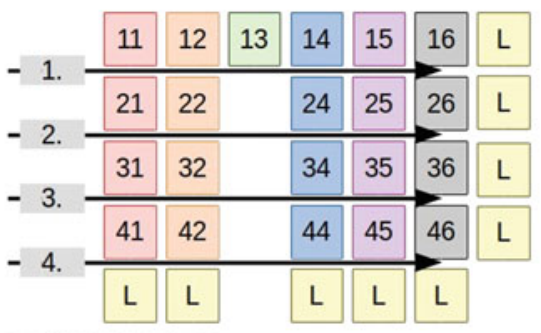

zeilenweise blockweise

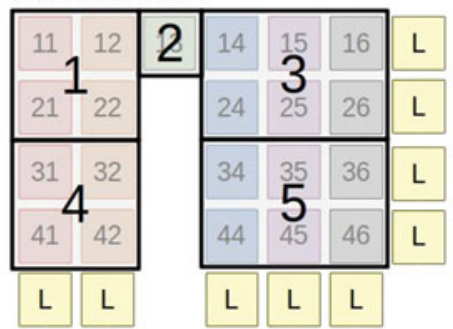

Abb.6.4 Varianten des inhaltlichen Unterrichtsablaufs

Das Beispiel in Abb. 6.2 könnte mit den Blöcken 11, 13, 14 und 21 bis 26 erarbeitet werden.

Wir betrachten als ein konkretes Beispiel den Ablauf durch die dritte Zeile. Abb. 6.5 zeigt eine Übersicht dazu, inklusive einer App-Aufgabenstellung.

Vorgehensweise: Ein bearbeitetes Bild ist gegeben. Die erste Aufgabe besteht darin, im Bild den zu modellierenden Prozess zu erkennen. Hier ist es eine anisotrope Diffusion mit diagonaler, von links unten nach rechts oben, Vorzugsrichtung. Das passende Modell[32] beinhaltet Richtungsableitungen zweiter Ordnung ${ }^{[31]}$. Darin enthalten ist die Hessematrix. Block [13] beinhaltet die Behandlung eines digitalen Bildes als diskrete Funktion, für die die Hessematrix ${ }^{[34]}$ aufgestellt werden muss. Die Hessematrix als diskreter Ableitungsterm lässt sich mit $3 \times$ 3-Filtern $-F_{x x}, F_{x y}$ und $F_{y y}$ - darstellen. Das zugehörige diskrete Modell ${ }^{[35]}$ führt dann auf eine Linearkombination der berechneten Filter. Dort angekommen ist man in der Lage, die Matrix- und Parameterfelder der App korrekt auszufüllen. Nach Anwendung auf das entsprechende Originalbild kann durch einen Bildvergleich eine qualitative Beurteilung erfolgen. Es ist unwahrscheinlich, dass man 


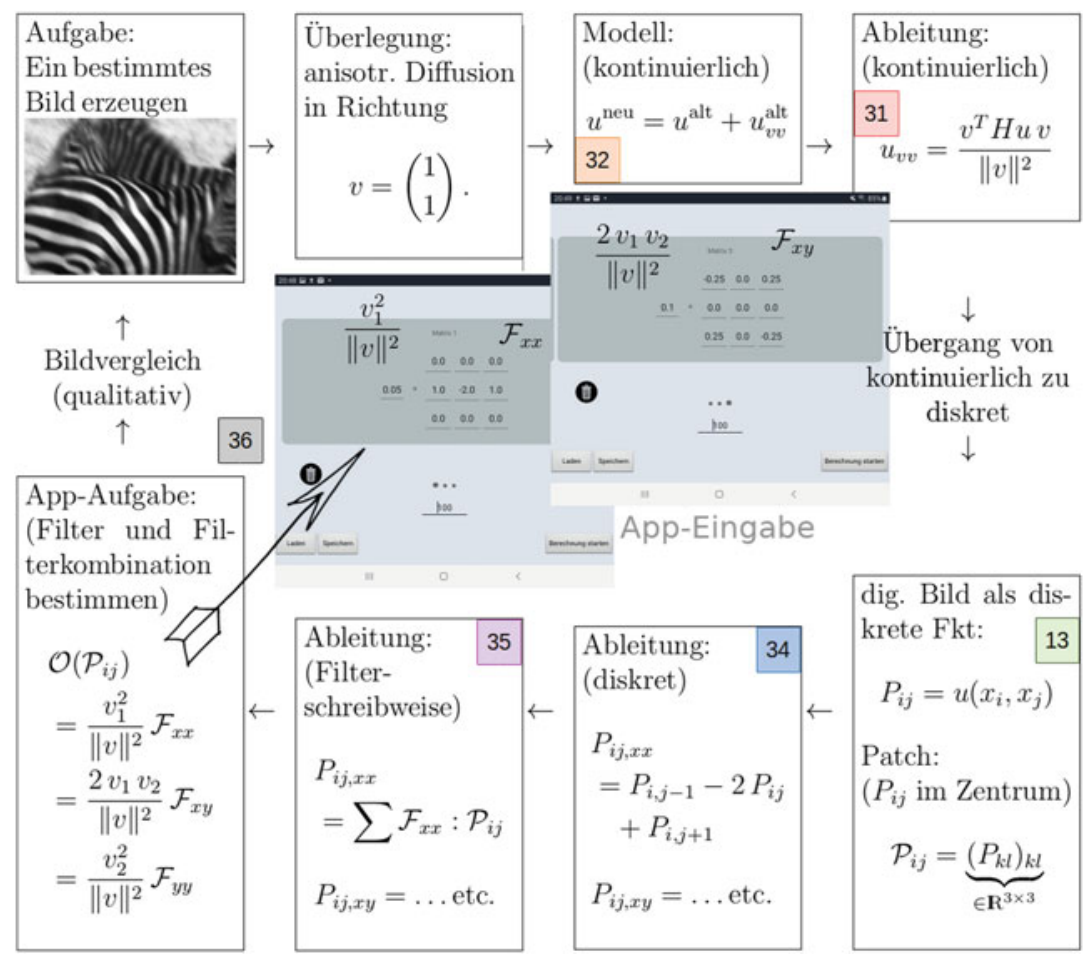

Abb.6.5 Beispiel einer Aufgabenstellung mit Richtungsableitungen

zufällig korrekte Werte angibt. Die App lässt sich ohne vorab erarbeitetes Wissen nicht bedienen.

\subsection{Erkenntnisse}

Es wurden zwei Evaluationen in je zwei Schritten durchgeführt: Jeweils eine Preund eine Post-Befragung vor und nach der Durchführung dieser Lehreinheit. Die erste Evaluation fand im Sommersemester 2019, die zweite im Sommersemester 2020 statt. Die Evaluationen erlauben einen Einblick, sowohl in die Entwicklung jeweils einer Studierendengruppe (Pre- und Posttest), als auch in die Entwicklung des Unterrichtskonzepts selbst (zwei verschiedene, befragte Gruppen). Bei 
der Pre-Befragung 2019 bzw. 2020 nahmen $56.45 \%$ (35 von 62) bzw. $48.93 \%$ (15 von 31) teil und bei der Post-Befragung lag die Beteiligung 2019 bzw. 2020 bei $40.32 \%$ ( 25 von 62 ) bzw. $22.58 \%$ (7 von 31). Die niedrigere Beteiligung bei der Post-Befragung 2020 lässt sich dadurch erklären, dass diese Unterrichtseinheit zum Ende des Semesters erfolgte, welches durch die Corona-Pandemie verkürzt war und dadurch alle gegen Ende in eine verstärkte Zeitnot brachte. Die Studierenden richteten ihre Aufmerksamkeit zunehmend auf die anstehenden Prüfungen aus.

Bei der erstmaligen Durchführung des Kapitels wurde die Matrix zeilenweise durchlaufen (siehe Abb. 6.4). Das hatte zu einem Vermischen von Vorlesungsund Übungsstrukturen geführt. Die Vorlesung war stärker durchsetzt mit Saalaufgaben, was die Studierenden sehr begrüßt hatten, dafür musste in der Übung ein wenig Lehrmaterial erarbeitet werden. Diese Unterrichtsweise funktioniert allerdings nur, wenn alle Beteiligten ,am Ball“ bleiben. Die Teilnehmer sind gewohnt, dass sie in der Übung auch ältere Aufgaben bearbeiten oder ein wenig Vorlesungsinhalte nacharbeiten können. Das didaktische Konzept dieses letzten Kapitels forderte ein gleichzeitiges Vorankommen. Ein Kommentar der Evaluation bringt es auf den Punkt: ,Interessante, neue Unterrichtsmethodik $=>$ stärker angeleitet". Die positiven Kommentare beinhalten hauptsächlich die Stichworte „,interessant" und ,gutes, hilfreiches Arbeitsmaterial" und werden mit 25 von 35 mehr als doppelt so häufig erwähnt als die negativen Kommentare, die sich hauptsächlich auf den "Zeitfaktor" und „Komplexität des Themas" beziehen. Weitere Kommentare sind als neutral einzustufen oder beziehen sich auf spezielle Situationen im Semester, die unabhängig sind von den Inhalten und dem Konzept dieser Lehreinheit. Das ist ein zunächst zufriedenstellendes Ergebnis. Dennoch zeigt die Tab. 6.1 (2019-A), dass nach der Unterrichtseinheit mehr Studierende das Problem „Das theoretische Wissen in der Praxis anwenden“ stark gewichtet haben. Die Nahtstelle Theorie-Praxis ist im Laufe des Unterrichts demnach noch sichtbarer geworden.

Beim zweiten Durchlauf wurde, der Zeitersparnis wegen, ein blockweiser Weg (Abb. 6.4, links unten) gewählt. Den Studierenden sollte wieder mehr Raum zum zeitlich selbstbestimmten Lernen gegeben werden, weshalb zur Unterstützung als Lehrmaterial verstärkt Tutorials eingesetzt wurden. Tab. 6.1 (2019-B) zeigt, dass die Studierenden deutliche Schwierigkeiten mit dem Lehrmaterial hatten. Nach dem zweiten Durchgang (2020-B) hat dies niemand mehr als Problem angesehen. Der Anteil positiver Kommentare zur Gesamtzahl der Kommentare, die sich auf diese spezielle Lehreinheit beziehen lassen, entspricht ungefähr dem im ersten Durchgang. Insgesamt lässt sich sagen, dass die Studierenden sehr am Thema interessiert waren. Sie konnten auch die Brücke zwischen Theorie und Praxis 
Tab.6.1 Vergleich Pre-/Post-Befragung (Ein hoher Rang bedeutet eine niedrige Gewichtung des genannten Problems und umgekehrt)

F: "Welche der folgenden Probleme sind im Rahmen der Veranstaltung aufgetreten?"

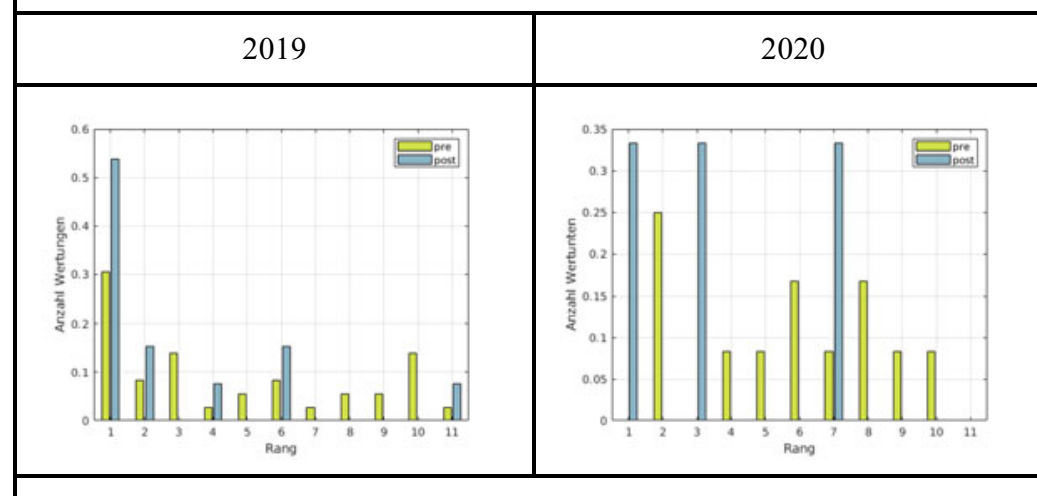

A: "Das theoretische Wissen in der Praxis anwenden"

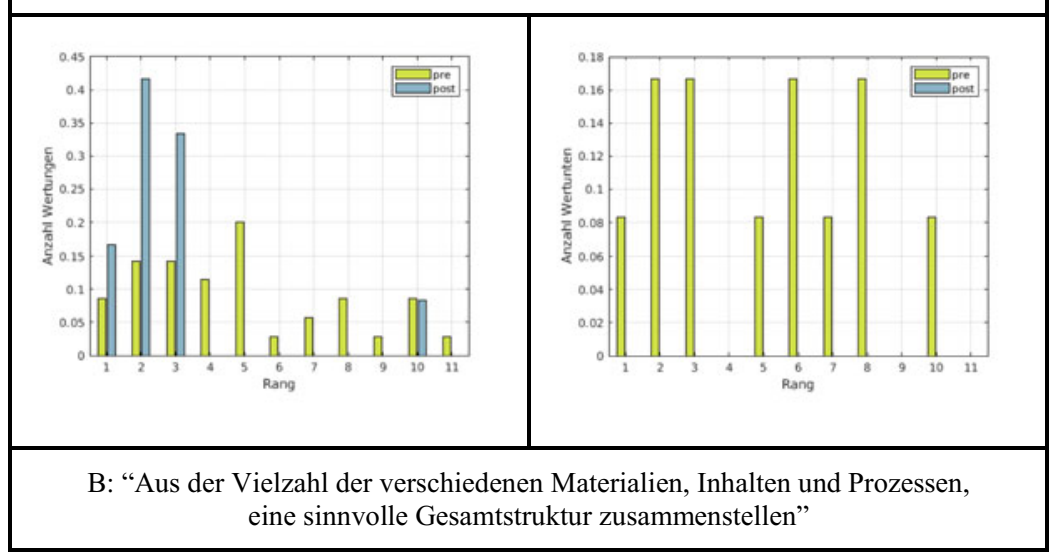

erkennen, konnten diese auch einmal betreten aber sie waren nicht in der Lage, sie selbstständig zu überqueren. Die in Tab. 6.1 (2020-A) dargestellte Auswertung zeigt auch hier eine Verstärkung des Bruchs „Theorie-Praxis“, wenn auch nicht so drastisch wie im Jahr zuvor; aber dennoch vergleichbar. Tab. 6.2 zeigt, dass 
Tab.6.2 Auswertungen aus den Postumfragen 2019 und 2020

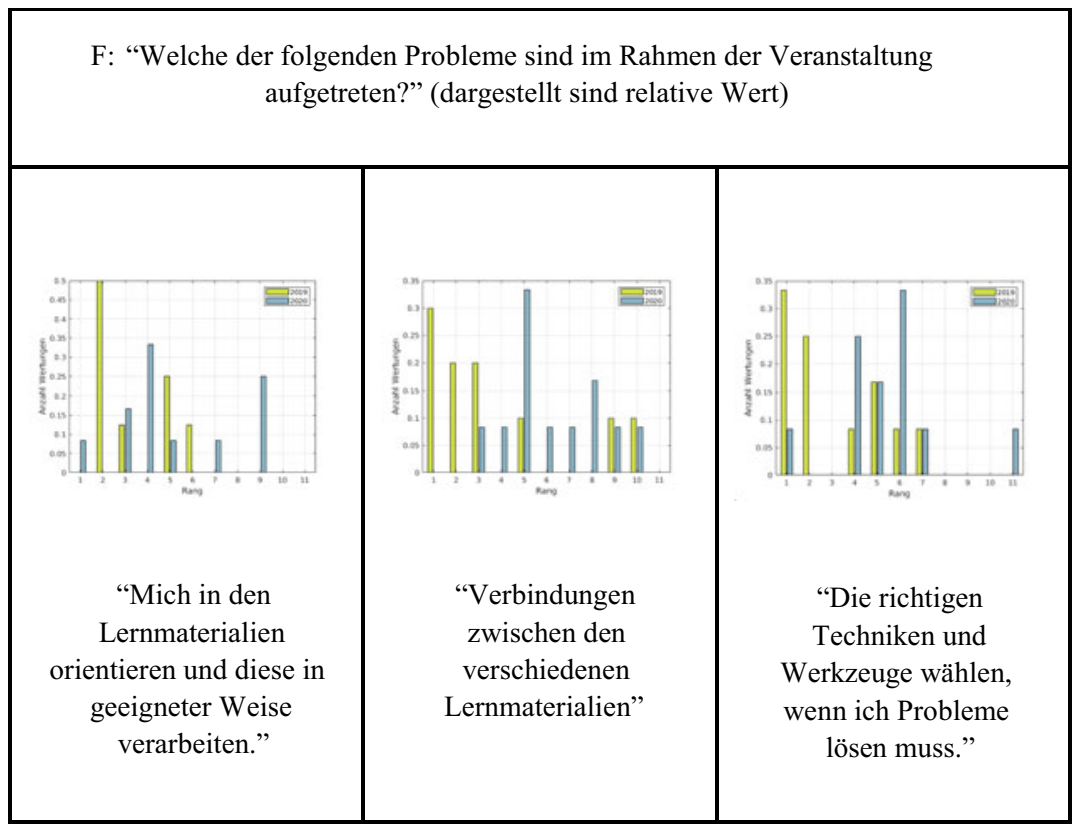

einige Problemstellungen im zweiten Durchgang deutlich besser bewertet wurden. Daraus lässt sich schließen, dass die Unterrichtsgestaltung im zweiten Gang didaktisch verbessert werden konnte.

Aus den Erfahrungen heraus hat ein ganzheitliches Konzept der Vorlesung hohe Priorität erhalten. So lange nicht die komplette Veranstaltung mit dem Matrixkonzept gestaltet werden kann, werden die Themenblöcke derart mit der Vorlesung verzahnt, dass für Studierende kein Bruch im Unterrichtsstil stattfindet. Beim nächsten Durchgang dieser Einheit wird auf sehr viel mehr Begleitung gesetzt werden. Das Material, das verschiedene inhaltliche Wege erlaubt wird bis auf Weiteres als zusätzliches Lernmaterial ausgehändigt.

\section{$6.4 \quad$ Fazit}

Wir sehen die Matrixstruktur im Lehrkonzept und die damit verbundene Möglichkeit, die Inhalte auf vielfältige Weise erarbeiten zu können, als ein innovatives 
Konzept mit großem Erfolgspotenzial an, um Theorie und Praxis von der ersten Stunde an näher zusammenzubringen.

Das größte Problem bei diesem Lehrkonzept war der Konzeptwechsel im Laufe der Gesamtveranstaltung. In der Tat empfehlen wir die komplette Lehrveranstaltung in dieser Art zu konzipieren, da sonst ein neuer Bruch entsteht, nämlich der Bruch des Lehrkonzepts innerhalb einer Vorlesung. Inhaltlich ist dieses Kapitel äußerst facettenreich und es ist daher dringend notwendig die Aufgaben zur App eng mit der Vorlesung zu verzahnen. Gelingt dies, so können die Studierenden schnell etwas "sehen“ und sind dann sehr motiviert, das Thema nicht nur oberflächlich zu erfassen. Dies gilt natürlich besonders für angehende Informatiker und Informatikerinnen. Da digitale Bildverarbeitung auch außerhalb der Informatik ein relevantes Thema ist sind die Inhalte auch für andere interessant.

Die erste Spalte beinhaltet mathematische Definitionen der beteiligten Ableitungsterme erster und zweiter Ordnung, welche in verschiedenen Modellen, die in der zweiten Spalte erklärt sind, benötigt werden. Vierte und fünfte Spalte wiederholen diese Themen für diskrete Funktionen. Der Übergang von kontinuierlichen zu diskreten Funktionen und der Zusammenhang zu digitalen Bildern ist in der dritten Spalte erklärt. Die sechste Spalte enthält Aufgaben, die mit der App bearbeitet werden können. 
Open Access Dieses Kapitel wird unter der Creative Commons Namensnennung 4.0 International Lizenz (http://creativecommons.org/licenses/by/4.0/deed.de) veröffentlicht, welche die Nutzung, Vervielfältigung, Bearbeitung, Verbreitung und Wiedergabe in jeglichem Medium und Format erlaubt, sofern Sie den/die ursprünglichen Autor(en) und die Quelle ordnungsgemäß nennen, einen Link zur Creative Commons Lizenz beifügen und angeben, ob Änderungen vorgenommen wurden.

Die in diesem Kapitel enthaltenen Bilder und sonstiges Drittmaterial unterliegen ebenfalls der genannten Creative Commons Lizenz, sofern sich aus der Abbildungslegende nichts anderes ergibt. Sofern das betreffende Material nicht unter der genannten Creative Commons Lizenz steht und die betreffende Handlung nicht nach gesetzlichen Vorschriften erlaubt ist, ist für die oben aufgeführten Weiterverwendungen des Materials die Einwilligung des jeweiligen Rechteinhabers einzuholen. 